\title{
Gametocitemia de Plasmodium falciparum según la respuesta terapéutica a sulfadoxina-pirimetamina y cloroquina en dos municipios de Antioquia, Colombia
}

\author{
Eliana Arango ${ }^{1}$, Tania Álvarez ${ }^{1}$, Jaime Carmona ${ }^{1}$, Silvia Blair ${ }^{1,2}$ \\ Grupo de Malaria, Universidad de Antioquia, Medellín, Colombia.
}

Se ha informado que el número de gametocitos circulantes de Plasmodium falciparum está influenciado por aspectos como el nivel de endemicidad de la zona, la clase de esquizonticidas sanguíneos usados y la respuesta terapéutica a ellos. En Colombia son muy pocos los trabajos que han evaluado estas relaciones. Mediante un diseño experimental, se evaluó la gametocitemia (variable efecto) en función del nivel endémico de dos municipios de Antioquia, del tratamiento (sulfadoxina-pirimetamina y sulfadoxina-pirimetamina más cloroquina) y de la respuesta terapéutica (adecuada y fallida). Se estudiaron 148 pacientes con malaria por $P$. falciparum no complicada. La gametocitemia varía en función del tiempo de padecimiento de la malaria actual (mayor en Turbo que en Zaragoza) y esta variable debe controlarse para eliminar la aparente diferencia en las gametocitemias por municipio. No se hallaron diferencias estadísticamente significativas en la gametocitemia (porcentaje de pacientes con gametocitos circulantes y cantidad de ellos por microlitro) según el tratamiento y la respuesta terapéutica, aunque los niveles de gametocitos son mayores en los pacientes tratados sólo con sulfadoxinapirimetamina, respecto a quienes recibieron sulfadoxina-pirimetamina más cloroquina. Tampoco hubo diferencias en la gametocitemia según el sexo ni la edad de los pacientes, ni se halló correlación de ella con la parasitemia asexual. La diferencia en el nivel de gametocitemia encontrada entre los municiios de Turbo y Zaragoza parece estar influida por el tiempo transcurrido entre el inicio de los síntomas y la instauración del tratamiento.

Palabras clave: gametocitemia, Plasmodium falciparum, Turbo, Zaragoza, cloroquina, sulfadoxina-pirimetamina, respuesta terapéutica.

Gametocyte levels in response to differing malaria treatments in two municipalities of Colombia

Plasmodium falciparum gametocyte levels are influenced by level of regional endemicity, the antimalarial treatment, and the therapeutic response of patients. Few previous studies have related these factors in Colombia. Here, gametocytaemia was evaluated with respect to two treatment schemes (sulfadoxine/pyrimethamine and sulfadoxine/pyrimethamine plus chloroquine), the patient response (adequate or failure), and the locality (two areas of varying case frequency). One hundred forty-eight residents of Turbo and Zaragoza (Antioquia), all with uncomplicated malaria, were evaluated. The gametocytaemia and the rates of clinical malaria at the beginning of treatment were greater in Turbo than in Zaragoza. No statiscally significant differences in the gametocytaemia by treatment schemes or therapeutic responses were noted, although the patients who received SP had more gametocytes than those treated with $S P+C Q$. Gametocytaemia was not correlated with asexual parasitemia or sex and age of patient. The difference in the level of gametocytaemia between Turbo and Zaragoza appears to be influenced by the time elapsed between the appearance of symptoms and the beginning of treatment.

Key words: gametocytaemia, P. falciparum, Turbo, Zaragoza, chloroquine, sulfadoxinepyrimethamine, therapeutic responses 
Los gametocitos son células sexuales altamente diferenciadas que se producen durante el ciclo eritrocítico de los parásitos del género Plasmodium y son necesarias para la continuidad del ciclo de vida en el mosquito vector (1-3). Deben ser transmitidos del hospedero vertebrado a su vector y el éxito de la transmisión depende de la disponibilidad de gametocitos infectivos circulantes en el vertebrado. Se ha observado una correlación positiva entre la gametocitemia y el riesgo de transmisión de la malaria en diferentes poblaciones (1-5).

En la reproducción de Plasmodium falciparum, después de varios ciclos de proliferación asexual, una pequeña porción de parásitos se diferencia a gametocitos al final de un proceso de cinco estadios morfológicamente diferentes cuya maduración completa se alcanza entre 10 y 12 días; dicho proceso se conoce como gametocitogénesis (1-3). Los estadios tempranos de los gametocitos (I-IV) permanecen secuestrados en la microcirculación periférica del hospedero humano aproximadamente por siete días, mientras que los maduros se mantienen en la fase $G_{0}$ del ciclo celular y circulan en sangre periférica hasta que son tomados por el vector $(1,3,6)$. Estas células pueden permanecer en la circulación del hospedero entre tres y ocho semanas $(1,5,7)$.

Diversos estudios han informado que el número de gametocitos circulantes en $P$. falciparum está influenciado tanto por aspectos del hospedero (la respuesta inmune), del parásito (la cantidad de parásitos asexuales y su capacidad para diferenciarse a gametocitos), como por aspectos externos (el tipo de medicamento suministrado en el control de los estadios asexuales) (1,3,621). Además, algunos estudios muestran que los pacientes con falla al tratamiento antimalárico o aquéllos que requieren más tiempo para eliminar los parásitos asexuales, presentan mayor número de gametocitos circulantes que los pacientes que responden adecuadamente al tratamiento o cuya

Correspondencia:

Sylvia Blair, Universidad de Antioquia, Carrera 51D ํ62-29, piso 3, Medellín, Colombia.

Teléfono: 510 6058; fax: 2633509

sblair@catios.udea.edu.co

Recibido: 23/04/03; aceptado: 27/02/04 parasitemia asexual desaparece más rápidamente con el tratamiento suministrado $(11,17,18)$. Sin embargo, hay datos contradictorios acerca de la influencia que tienen los esquizonticidas sanguíneos sobre la gametocitemia de pacientes con malaria por $P$. falciparum $(7,12-15,17,19)$.

La mayoría de los estudios que buscan relacionar la endemicidad por malaria y el número de gametocitos se han llevado a cabo en regiones africanas holoendémicas para malaria (22). En Colombia, las regiones antioqueñas de Urabá y el Bajo Cauca son consideradas de alto riesgo de transmisión para malaria y corresponden a áreas de baja endemicidad según la Organización Mundial de la Salud (OMS) (22). En las localidades de Turbo y Zaragoza, el índice parasitario anual (IPA*) promedio observado entre 1996 y 2001 presentó una diferencia significativa (Turbo: 38,86 y Zaragoza: 161,75) (datos suministrados por la Dirección Seccional de Salud de Antioquia, Departamento de Epidemiología). Esto sugiere que estas dos regiones tienen riesgos diferentes para la infección malárica. Los estudios realizados por nosotros en 1998 confirmaron la existencia de falla terapéutica al tratamiento con cloroquina en Turbo y Zaragoza en un $97 \%$ y $67 \%$, respectivamente, mientras que al tratamiento con sulfadoxina/ pirimetamina se observó en un $9 \%$ y $13 \%$, respectivamente $(23,24)$.

Este trabajo evaluó las diferencias en la gametocitemia en función de la localidad, el tratamiento y la respuesta terapéutica en pacientes con malaria no complicada por $P$. falciparum residentes en los municipios de Turbo y Zaragoza que consultaron al puesto de malaria durante el primer semestre de 2002.

\section{Materiales y métodos}

\section{Población de estudio}

El estudio se realizó en los municipios de Turbo y Zaragoza (departamento de Antioquia) entre febrero y abril de 2002. Se incluyeron pacientes voluntarios con diagnóstico de malaria no complicada por $P$. falciparum que consultaron al puesto de malaria en cada una de las dos localidades. Se utilizaron los criterios de inclusión

*IPA: número de casos anuales nuevos por cada 1.000 habitantes expuestos a la malaria 
y exclusión del protocolo de evaluación de la respuesta terapéutica a los antimaláricos (25).

Tipo de estudio y selección de la muestra

Se usó un diseño experimental de grupos balanceados, en el que cada paciente con malaria fue sometido a tratamiento con uno de tres esquemas terapéuticos, a saber: 1) cloroquina (CQ); 2) sulfadoxina/pirimetamina (SP), y 3) combinado: CQ más SP. Se calculó el tamaño de la muestra para proporciones de poblaciones finitas (26) con la siguiente ecuación:

$$
n=\frac{N^{*} Z^{2 *} p(1-p)}{\left(N^{*} e^{2}\right)+\left(Z^{2 *} p(1-p)\right)}
$$

donde:

$\mathrm{n}$ : tamaño calculado de la muestra

$\mathrm{N}$ : población de pacientes maláricos con $P$. falciparum, que consulta al puesto de malaria (personas de cualquier sexo, toda edad y demás características demográficas y epidemiológicas)

Z: nivel de confianza que se fija en el $95 \%$, lo que implica un error alfa (nivel de significación) de $5 \%$.

p: proporción esperada de presentación del evento de falla terapéutica

(1-p): complemento de $p$

e: error de muestreo, definido en $12 \%$.

Se calcularon los tamaños de la muestra para cada fármaco en cada municipio usando la información sobre frecuencia de malaria por $P$. falciparum en Turbo y Zaragoza en 1999, los datos conocidos de frecuencia de falla terapéutica en los dos lugares $(23,24)$, los valores de $Z$ y de "e" ya indicados y con una frecuencia de falla con la combinación CQ+SP obtenida como probabilidad combinada (producto) de falla para CQ y para SP; así llegamos a lo siguiente:

\begin{tabular}{lccllll}
\hline $\mathbf{N}$ & \multicolumn{1}{c}{$\mathbf{Z}$} & $\mathbf{p}$ & $\mathbf{1 - p}$ & $\mathbf{E}$ & $\mathbf{n}$ & Droga y lugar \\
\hline 595 & 1,96 & 0,97 & 0,03 & $12 \%$ & 8 & CQ-Turbo \\
466 & 1,96 & 0,67 & 0,33 & $12 \%$ & 52 & CQ-Zaragoza \\
595 & 1,96 & 0,13 & 0,87 & $12 \%$ & 29 & SP-Turbo \\
466 & 1,96 & 0,09 & 0,91 & $12 \%$ & 21 & SP-Zaragoza \\
595 & 1,9 & 0,126 & 0,874 & $12 \%$ & 28 & SP/CQ-Turbo \\
466 & 1,96 & 0,060 & 0,94 & $12 \%$ & 15 & SP/CQ-Zaragoza \\
\hline
\end{tabular}

Según lo anterior, el tamaño de la muestra osciló entre 8 y 52 , dependiendo del tratamiento y del lugar. Se decidió obtener un tamaño muestral promedio de 33, y ésta fue la muestra finalmente evaluada en cada lugar y con cada tratamiento. Los pacientes se asignaron aleatoriamente a uno de los tres esquemas y el tratamiento se administró de manera supervisada.

\section{Diagnóstico y recuento de formas parasitarias}

A cada paciente se le tomó gota gruesa que se coloreó con Field. El diagnóstico se realizó en 100 campos microscópicos y se hizo recuento de formas asexuales y de gametocitos por cada 200 leucocitos; se tomaron 8.000 leucocitos $/ \mu \mathrm{L}$ como la constante de leucocitos en la población. De esta manera, se calculó el número de parásitos por microlitro de sangre.

\section{Tratamiento y seguimiento de los pacientes}

Para el grupo de pacientes del esquema $C Q$, el tratamiento consistió en una dosis total de $25 \mathrm{mg}$ de cloroquina por $\mathrm{kg}$ de peso corporal dividida en tres días ( $10 \mathrm{mg} / \mathrm{kg}$ de peso el primer día, día 0 , y $7,5 \mathrm{mg} / \mathrm{kg}$ de peso en los dos días siguientes). Al grupo SP se le suministró sulfadoxina/ pirimetamina en dosis única de $25 \mathrm{mg} / 1,25 \mathrm{mg}$ por kg de peso; y el grupo $\mathrm{SP}+\mathrm{CQ}$ recibió terapia combinada con SP-25 mg/1,25 mg por $\mathrm{kg}$ de peso el primer día y los tres días siguientes $25 \mathrm{mg} / \mathrm{kg}$ de peso de $C Q$ dosificado como en el esquema $C Q$. La administración del tratamiento fue supervisada por los investigadores. Los pacientes se siguieron clínica y parasitológicamente los días $1,2,3,7,14$ y 21 después de iniciado el tratamiento. A los pacientes que presentaron falla al tratamiento, según los criterios de la OMS de 1998, se les suministró un tratamiento de rescate con quinina oral, $10 \mathrm{mg} / \mathrm{kg}$ cada 8 horas por 3 días, más clindamicina, $5 \mathrm{mg} / \mathrm{kg}$ de peso cada 6 horas durante 5 días.

En el último día de control, después de la toma de la gota gruesa, a todos los pacientes se les suministró una dosis de primaquina $(0,75 \mathrm{mg} / \mathrm{kg}$ de peso), con el fin de que las variaciones en la gametocitemia no estuvieran afectadas por la primaquina, cuya actividad gametocida es conocida. 
Definición de la variable efecto (gametocitemia) $y$ de las variables explicativas

El efecto consiste en la gametocitemia expresada tanto como pacientes con gametocitos circulantes como concentración de estas células por microlitro de sangre.

Se definen también estos términos:

- Gametocitemia diaria: la cantidad de gametocitos por microlitro en un día determinado.

- Gametocitemia total: cantidad de gametocitos acumulada desde el día 1 hasta el 21; es la suma de los gametocitos diarios.

- Gametocitemia total promedio: cantidad de gametocitos acumulada desde el día 1 hasta el 21 dividida por el número de mediciones (que fue de 7). Expresa la masa de gametocitos producida.

Las variables explicativas consideradas en este trabajo son tres: 1) municipio, busca expresar la influencia del nivel endémico de malaria; 2) el medicamento usado (SP o $S P+C Q)$, y 3) la respuesta terapéutica observada.

Durante el análisis de los datos, se encontró que el tiempo de evolución de la enfermedad podía actuar como una variable de confusión y se procedió a evaluar su influencia sobre la variable efecto.

La respuesta terapéutica se clasificó según los criterios de la OMS (25), así:

Falla terapéutica precoz:

- Signos de peligro o malaria grave los días 1,2 o 3.

- Parasitemia del día 2 mayor a la del día 0.

- Parasitemia del día $3 \geq 25 \%$ del recuento del día 0.

Falla terapéutica tardía:

- Signos de peligro o malaria grave después del día 3 con parasitemia.

- Regreso no programado del paciente entre los días 4 a 21 debido al deterioro clínico con parasitemia.
- Presencia de parasitemia (de la misma especie que el día 0) en los días 7, 14 o 21.

Respuesta clínica adecuada:

Si el paciente no presenta ningún criterio de falla terapéutica temprana o tardía y la gota gruesa permanece negativa a partir del día 4 y hasta el último control el día 21.

\section{Consideraciones éticas}

Este proyecto fue aprobado por el Comité de Ética del Centro de Investigaciones Médicas de la Facultad de Medicina de la Universidad de Antioquia. A los individuos que cumplieron los criterios de inclusión se les informó sobre los objetivos, los riesgos y los beneficios del estudio y, luego de aceptar su participación en él, firmaron el consentimiento informado.

\section{Análisis estadístico}

El análisis de los datos se realizó en el programa estadístico Epi-Info, versión 6.04. Los valores de tendencia central de las variables (medianas) se compararon entre grupos con la prueba de Kruskal y Wallis, mientras que las distribuciones de frecuencias se analizaron con la prueba de ji al cuadrado. Se buscaron asociaciones entre variables métricas mediante el análisis de correlación-regresión lineal simples de Pearson. Siempre se usó un nivel menor de $5 \%$ para declarar como significativa una probabilidad.

La media aritmética se expresa como $\bar{X}$; la mediana, Me, y la desviación estándar, DE.

\section{Resultados}

Hallazgos generales y características de la población de estudio

Se estudiaron 148 pacientes, 72 en Turbo y 76 en Zaragoza, con edades que oscilaron entre 1 y 74 años (promedio: $24 \pm 17$ ). El porcentaje de hombres fue de $58 \%$ y $66 \%$ en Turbo y Zaragoza, respectivamente. El tiempo de evolución de la enfermedad antes del inicio del tratamiento osciló entre 1 y 30 días en Turbo (promedio \pm DE: $5 \pm 6$ días) y entre 1 y 15 días en Zaragoza ( $4 \pm 3$ días). En Turbo, el $72 \%$ de los pacientes tenía hasta 5 días de evolución de la malaria cuando ingresó al estudio, mientras que en Zaragoza el $85 \%$ de los 
pacientes presentaron esta característica. Los promedios aritméticos con sus DE de la parasitemia asexual inicial fueron de $4.661 \pm 5.967$ y $5.694 \pm 5.372$ anillos/ $\mu$ l en Turbo y Zaragoza, en su orden $(p<0,001)$. En cualquier día - desde el ingreso al estudio (día 0) hasta el día 21- la correlación lineal entre gametocitemia y parasitemia asexual presentó un coeficiente $r<0,1$.

Diecisiete pacientes recibieron $C Q, 67$ recibieron $\mathrm{SP}$ y 64 el esquema combinado SP+CQ. Cuando se completaron 17 pacientes tratados con $C Q, 8$ en Turbo y 9 en Zaragoza, el $82 \%$ ( $n=14)$ había presentado falla terapéutica; por tal razón, se suspendió la administración de este tratamiento sin alcanzar los 33 pacientes que se habían calculado para la muestra y se excluyó este grupo de pacientes para el análisis de los resultados.

\section{Comportamiento de la gametocitemia en el tiempo, por municipio}

La diferencia en la concentración de gametocitos por microlitro de sangre entre los pacientes que presentaban antecedentes de malaria en el último año y aquéllos que no los tenían, no fue estadísticamente significativa. Tampoco se encontraron diferencias significativas en la gametocitemia con respecto a la edad o el sexo.

Al iniciar el estudio (día de diagnóstico), el 22\% de los pacientes de Turbo y el 7\% de Zaragoza tenía gametocitos circulantes. El día 3, los pacientes con gametocitos en sangre eran $49 \%$ en Turbo y $40 \%$ en Zaragoza, mientras el día 7 los valores eran de $94 \%$ y $92 \%$, respectivamente. La gametocitemia diaria y total promedio por municipio es significativamente mayor $(p<0,05)$ en Turbo que en Zaragoza en cualquier día, excepto el día 21, cuando es mayor en Zaragoza y la diferencia no es significativa (cuadro 1, figura 1).

Como el tiempo de evolución de la malaria es mayor en Turbo que en Zaragoza y, por ende, puede afectar la gametocitemia, actuando como una variable de confusión, se procedió a examinar la influencia de esta variable en la cantidad de gametocitos por municipio y se midió en cada lugar de acuerdo con determinado tiempo de evolución de la enfermedad. Los pacientes con 1 , 2 y 5 días de evolución de su enfermedad carecen de gametocitos en ambos municipios. Aquéllos con 3 días de evolución presentan gametocitos en $15 \%$ y $17 \%$ de las veces en Turbo y Zaragoza, en su orden, mientras que el día 4, los valores son de $8 \%$ y $6 \%$, respectivamente. La cantidad de gametocitos en los días 3 y 4 es estadísticamente igual en cada municipio (Turbo: día 3:

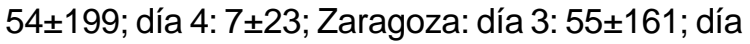
4: $2 \pm 10)$. En resumen, la diferencia en la gametocitemia diaria y promedio total entre municipios es similar cuando la comparación se hace controlando la influencia de la variable días de evolución de la malaria. Además, el análisis de correlación lineal simple entre la cantidad de gametocitos y los días de evolución de la enfermedad demuestra que en ambos lugares tal asociación es débil (coeficiente $r=0,37$ a 0,45) y carece de significación estadística.

Cuadro 1. Frecuencia de pacientes con gametocitemia y gametocitemia diaria por municipio y día de observación.

\begin{tabular}{|c|c|c|c|c|c|c|}
\hline \multirow[b]{2}{*}{ Día } & \multicolumn{3}{|c|}{ Turbo } & \multicolumn{3}{|c|}{ Zaragoza } \\
\hline & $\mathrm{n}$ & $\%$ & 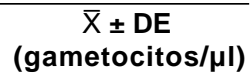 & $\mathbf{n}$ & $\%$ & $\begin{array}{c}\bar{X} \pm \mathrm{DE} \\
\text { (gametocitos/ } \mu \mathrm{l} \text { ) }\end{array}$ \\
\hline 0 & 64 & 22 & $66 \pm 200$ & 67 & 7 & $6 \pm 36$ \\
\hline 1 & 64 & 25 & $66 \pm 150$ & 63 & 18 & $14 \pm 63$ \\
\hline 2 & 64 & 36 & $126 \pm 268$ & 65 & 28 & $35 \pm 175$ \\
\hline 3 & 61 & 49 & $163 \pm 348$ & 64 & 39 & $77 \pm 364$ \\
\hline 7 & 61 & 94 & $613 \pm 695$ & 52 & 25 & $314 \pm 494$ \\
\hline 14 & 60 & 97 & $408 \pm 410$ & 48 & 26 & $292 \pm 505$ \\
\hline 21 & 54 & 86 & $181 \pm 236$ & 58 & 24 & $213 \pm 579$ \\
\hline GTP & 61 & 95 & $229 \pm 413$ & 60 & 88 & $122 \pm 380$ \\
\hline
\end{tabular}

$\mathrm{n}$ : número de pacientes con gametocitos circulantes; \%: porcentaje de pacientes con gametocitos circulantes; $\overline{\mathrm{X}}$ : promedio; DE: desviación estándar; GTP: gametocitemia total promedio 


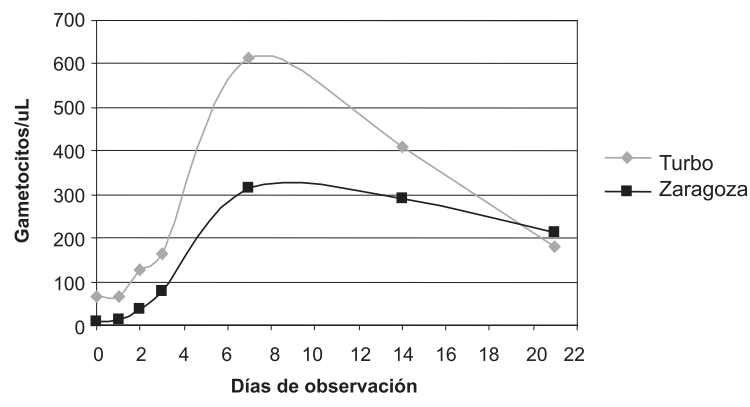

Figura 1. Comportamiento de la gametocitemia durante el período de observación, por municipio.

En general, las curvas de comportamiento de la gametocitemia en el tiempo en Turbo y Zaragoza son similares al principio y al final del período de observación, pero en el intermedio son francamentes distintas, debido a los valores significativamente mayores encontrados en los pacientes de Turbo entre los días 4 a 14 (figura 1).

Gametocitemia en el tiempo según tratamiento y respuesta terapéutica

En cada tratamiento (SP y $\mathrm{SP}+\mathrm{CQ}$ ), la respuesta terapéutica fue similar en ambos municipios (cuadro 2, parte A) por lo cual en adelante se omite la presentación de datos según la variable municipio.
La respuesta terapéutica entre los tratamientos administrados sí fue muy diferente, debido a que la falla estuvo más frecuentemente asociada acon el tratamiento con SP únicamente (cuadro 2, parte B).

La frecuencia diaria de pacientes con gametocitos circulantes de acuerdo con cada grupo de tratamiento y la respuesta terapéutica se presenta en el cuadro 3 , parte $A$, en el cual se aprecia que no existe asociación significativa entre las variables grupo y presencia de gametocitos. Tampoco se halló diferencia significativa entre los valores de gametocitos de los seis grupos considerados ni entre los dos tratamientos (SP vs. $\mathrm{SP}+\mathrm{CQ}$ ) (cuadro 3, parte $\mathrm{B}$, figura 2).

\section{Discusión}

Nuestros hallazgos de ausencia de relación entre la gametocitemia y la edad o el sexo no concuerdan con otros informes $(10,14,16)$ y no tenemos explicación alguna para presentar.

Se ha encontrado una correlación positiva entre la gametocitemia y el riesgo de transmisión de la malaria en diferentes poblaciones africanas y asiáticas (2,3,9-11), de tal forma existe una relación directa entre el nivel de endemicidad y la gametocitemia: a mayor endemia, mayor gametocitemia. Entre 1996 y 2001, Turbo y

Cuadro 2. Respuesta terapéutica a los esquemas de tratamiento en cada municipio.

Parte A. Respuesta terapéutica entre municipios, en cada tratamiento

\begin{tabular}{|c|c|c|c|c|c|c|}
\hline & \multicolumn{2}{|c|}{ SP* $^{*}$} & \multirow[b]{2}{*}{$p$} & \multicolumn{2}{|c|}{$\mathrm{SP}+\mathrm{CQ}^{\star}$} & \multirow[b]{2}{*}{$p$} \\
\hline & Turbo & Zaragoza & & Turbo & Zaragoza & \\
\hline Adecuada & 26 (79\%) & 25 (74\%) & & 24 (77\%) & 29 (88\%) & \\
\hline Falla & $7(21 \%)$ & $9(26 \%)$ & 0,8 & 7 (23\%) & $4(12 \%)$ & 0,4 \\
\hline Total & 33 & 34 & & 31 & 33 & \\
\hline
\end{tabular}

* Expresado en número de pacientes y porcentaje

Parte B. Clasificación de la respuesta terapéutica de acuerdo con el esquema de tratamiento recibido.

\begin{tabular}{lcccr}
\multicolumn{2}{c}{ Respuesta terapéutica } & & Total \\
\hline Tratamiento & Falla precoz & Falla tardía & Adecuada & 67 \\
\hline SP & 9 & 7 & 51 & 64 \\
CQ+SP & 1 & 10 & 53 & 131 \\
Total & 10 & 17 & 104 & \\
\hline
\end{tabular}

$\chi^{2}=6,9 ; g \mathrm{l}=2, p=0,0317$ 
Cuadro 3. Frecuencia de pacientes con gametocitos circulantes y gametocitemia según el día de seguimiento y la respuesta terapéutica a cada tratamiento.

Parte A. Porcentaje de pacientes con gametocitos circulantes.

\begin{tabular}{|c|c|c|c|c|c|c|c|}
\hline \multirow[b]{2}{*}{ Día } & \multicolumn{3}{|c|}{ SP } & \multicolumn{3}{|c|}{$\mathrm{SP}+\mathrm{CQ}$} & \multirow[b]{2}{*}{$\mathbf{p}\left(\chi^{2}\right)$} \\
\hline & FP & FT & A & FP & FT & A & \\
\hline 0 & 0 & 29 & 14 & 0 & 40 & 13 & 0,17022119 \\
\hline 3 & 9 & 7 & 38 & 2 & 9 & 34 & 0,57572641 \\
\hline 7 & 7 & 6 & 39 & 0 & 7 & 39 & 0,83498892 \\
\hline 21 & 8 & 6 & 40 & 0 & 8 & 37 & 0,43121645 \\
\hline
\end{tabular}

Parte B. Gametocitemia (gametocitos $/ \mu \mathrm{l})^{\star}$; (promedio $\left.\pm \mathrm{DE}\right)$

\begin{tabular}{lccccccc}
\hline & \multicolumn{2}{c}{ SP } & & & \multicolumn{2}{c}{ SP+CQ } & \\
\cline { 2 - 4 } Día & FP & FT & & A & FP & FT & A \\
0 & 0 & $22 \pm 44$ & $55 \pm 209$ & & 0 & $31 \pm 48$ & $26 \pm 95$ \\
3 & $26 \pm 48$ & $121 \pm 236$ & $154 \pm 463$ &. & $119 \pm 171$ & $97 \pm 305$ \\
7 &.. & $506 \pm 515$ & $633 \pm 821$ &.. & $276 \pm 260$ & $353 \pm 404$ \\
21 &.. & $256 \pm 173$ & $194 \pm 254$ &.. & $71 \pm 70$ & $211 \pm 611$ \\
\hline
\end{tabular}

SP: sulfadoxina+pirimetamina; $\mathrm{SP}+\mathrm{CQ}$ : sulfadoxina+pirimetamina y cloroquina

FP: falla precoz; FT: falla tardía; A: respuesta clínica adecuada

* ninguna diferencia es estadísticamente significativa $(p>0,20)$;

.. indica que no hay pacientes en ese grupo

Zaragoza mostraron un IPA promedio de 39 por 1.000 y 162 por 1.000 , respectivamente. Contrario a lo esperado según la literatura, en este estudio se encontró una gametocitemia más alta en los pacientes de Turbo que en los de Zaragoza, tanto en el momento del diagnóstico como durante el seguimiento.

La gametocitemia también se podría ver afectada por el tiempo de duración de la malaria actual pues mientras mayor sea ésta, mayor será la posibilidad de que los gametocitos maduren y se observen en la sangre periférica. Los gametocitos de $P$. falciparum empiezan a aparecer en sangre periférica alrededor de una semana después de que aparecen los parásitos asexuales $(1,2)$, de donde puede deducirse que el tiempo de enfermedad influye en la gametocitemia. En nuestros pacientes, existe una diferencia de un día en el tiempo de evolución de la malaria actual entre Turbo y Zaragoza y aunque tal diferencia no fue estadísticamente significativa, ejerce influencia en la gametocitemia, como se demuestra al comparar la gametocitemia sin tener en cuenta el tiempo de evolución (hay diferencias significativas entre los dos municipios) y al compararla estratificando por tiempo de evolución (no hay diferencias significativas entre los dos municipios). Resulta, entonces, que la mayor frecuencia de pacientes con gametocitos y los mayores niveles de gametocitemia en Turbo están influenciados por el mayor tiempo de enfermedad que mostraron esos pacientes.

La diferencia en gametocitemia entre lugares también se ha explicado por la variabilidad en la constitución genética de los parásitos circulantes $(27,28)$. En Turbo y Zaragoza, este argumento tampoco podría aplicarse porque hemos demostrado que las poblaciones de parásitos circulantes en tales sitios son altamente homogéneas (29; Restrepo E, Villa AF, Maestre A, Blair S. Análisis molecular de las cepas de $P$. falciparum sensibles y resistentes a cloroquina y sulfadoxina-pirimetamina en Turbo y Zaragoza (Antioquia). Dirección Seccional de Salud de Antioquia y Universidad de Antioquia. 2002. En prensa).

También se ha dicho que la inmunidad adquirida por repetidas infecciones maláricas, como sucede en zonas de alta endemicidad, ayuda a controlar 

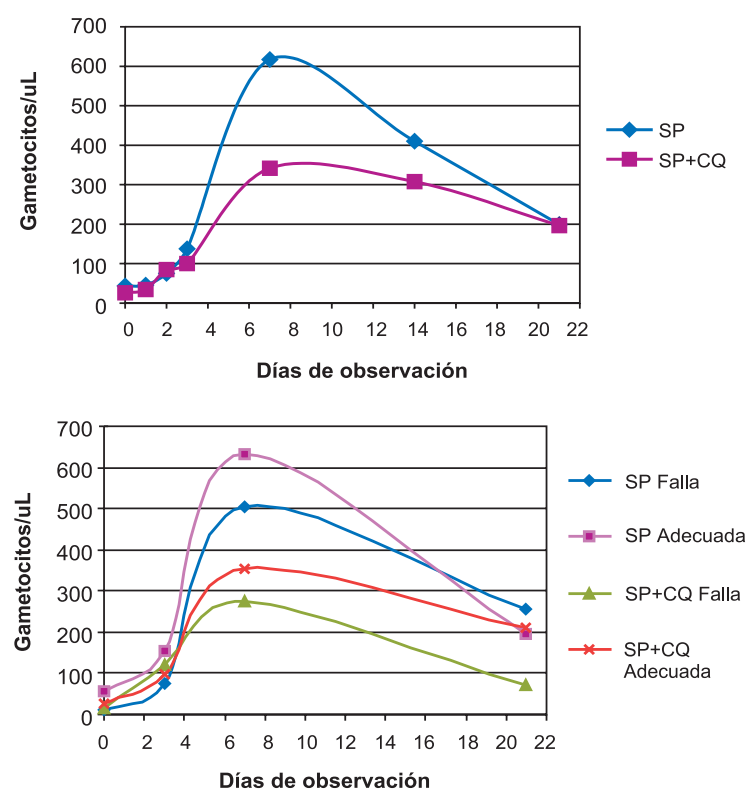

Figura 2. Evolución temporal de la gametocitemia según tratamiento (A) y grupo de tratamiento-respuesta terapéutica $(B)$.

las infecciones y a mantener bajos los niveles de parasitemia (1,2,30-32). Si Zaragoza tiene tres o más veces el nivel endémico de Turbo, se esperaría que allí la parasitemia asexual y la gametocitemia fuesen menores que en Turbo. La menor gametocitemia aparentemente sucedió, pero al controlar la influencia del tiempo de enfermedad, tal situación desapareció, mientras que la parasitemia asexual fue mayor en Zaragoza, contrario a lo supuesto.

Se ha informado que el tiempo transcurrido entre el inicio del tratamiento y la negativización de la gota gruesa es un factor que influye directamente la gametocitemia $(11,18)$; es decir, que los pacientes en quienes la eliminación de parásitos era más lenta (el tratamiento suministrado requería más tiempo para eliminar los parásitos circulantes), presentaron mayor cantidad de gametocitos en sangre periférica que los pacientes cuya eliminación de parásitos era más rápida. En el presente estudio no se encontró diferencia en la cantidad de gametocitos/microlitro entre los pacientes con respuesta adecuada (exitosa) y aquéllos con falla tardía, bien sea con SP o con $S P+C Q$, en ningún día de control.
El efecto de la CQ y la SP sobre la gametocitemia no está bien establecido. Los estudios realizados al respecto muestran resultados diferentes y, por tanto, cualquier conclusión debe hacerse con cautela $(7,13-17,19,20)$. En nuestro estudio encontramos que los pacientes que fueron tratados con SP presentaron gametocitemia durante todo el seguimiento (en especial entre los días 3 y 14) más altas que los pacientes tratados con $\mathrm{SP}+\mathrm{CQ}$, pero las diferencias no fueron estadísticamente significativas; esto concuerda con lo encontrado en algunos estudios $(15,17)$.

Los resultados de este estudio sugieren que, en Turbo y Zaragoza, la gametocitemia está afectada por el tiempo de duración de la enfermedad (relación directamente proporcional) pero no por el tratamiento aplicado (SP igual a $\mathrm{SP}+\mathrm{CQ}$ ) ni por la respuesta terapéutica a estos medicamentos (adecuada igual a falla). La relación entre endemia malárica y gametocitemia es inversa: hubo mayor gametocitemia en el sitio con menor IPA.

\section{Agradecimientos}

A la Dirección Seccional de Salud de Antioquia y a la Universidad de Antioquia por su apoyo financiero; al personal de los hospitales Francisco Valderrama de Turbo y San Rafael de Zaragoza; a los pacientes por su valiosa colaboración.

\section{Referencias}

1. Carter R, Graves PM. Gametocytes. En: Wernsdorfer WH, McGregor SI, editors. Malaria: principles and practice of malariology. First edition. Edinburgh, London, Melbourne and New York: Churchill Livingstone; 1988. p.253-99.

2. Fujioka $\mathbf{H}$, Aikawa $\mathbf{M}$. The malaria parasite and its lifecycle. En: Wahlgren M, Perlmann P, editors. Malaria: molecular and clinical aspects. First edition. Amsterdam: Harwood Academic Publishers; 1999. p.21-36.

3. Paul R, Brey P, Robert V. Plasmodium sex determination and transmission to mosquitoes. Trends Parasitol 2002;18:32-8.

4. Rogers NJ, Hall BS, Obiero J, Targett GA, Sutherland $\mathbf{C J}$. A model for sequestration of the transmission stages of Plasmodium falciparum: Adhesion or gametocyte-infected erythrocytes to human bone marrow cells. Infect Immun 2000;68:345562.

5. Robert V, Awono-Ambene HP, Le Hesran JY, Trape JF. Gametocytemia and infectivity to mosquitoes 
of patients with uncomplicated Plasmodium falciparum malaria attacks treated with chloroquine or sulfadoxine plus pyrimethamine. Am J Trop Med Hyg 2000; 62:210-6.

6. Shahabuddin M, Costero A. Spatial distribution or factors that determine sporogonic development of malaria parasites in mosquitoes. Insect Biochem Mol Biol 2001;31:231-40.

7. López FJ, Schmunis G. Diagnótico de malaria. Publicación científica No. 512. Washington, D.C.: OPS/ OMS; 1988.

8. Healer J, Graszynsky A, Riley E. Phagocytosis does not play a major role in naturally acquired transmissionblocking immunity to Plasmodium falciparum malaria. Infect Immun 1999;67:2334-9.

9. Buckling A, Read AF. The effect of partial host immunity on the transmission of malaria parasites. Proc R Soc Lond 2001;268:1-6.

10. Akim NIJ, Drakeley C, Kingo T, Dimon B, Senkoro K, Sauerwein W. Dynamics of $P$. falciparum gametocytemia in symptomatic patients in an area of intense perennial transmission in Tanzania. Am J Trop Med Hyg 2000;63:199-203.

11. Price R, Nosten F, Simpson JA, Luxemburger C, Phaipun L, Kuile FT, et al. Risk factors for gametocyte carriage in uncomplicated falciparum malaria. Am J Trop Med Hyg 1999;60:1019-23.

12. Seidlein L V, Jawara M, Coleman R, Doherty $\mathbf{T}$, Walraventul G, Targett G. Parasitaemia and gametocytaemia after treatment with chloroquine, pyrimethamine/sulfadoxine combined with artesunate in young Gambians with uncomplicated malaria. Trop Med Int Health 2001;6:1-7.

13. Strickland GT, Fox E, Sarwar M, Khaliq AA, Macdonald M. Effects of chloroquine, amodiaquine and pyrimethamine-sulfadoxine on Plasmodium falciparum gametocytemia. Am J Trop Med Hyg 1986; 35:259-62.

14. von Seidlein LV, Drakeley C, Greenwood B, Walraven G, Targett G. Risk factors for gametocyte carriage in Gambian children. Am J Trop Med Hyg 2001; 65:523-7.

15. Hogh B, Thompson R, Hetzel C, Fleck SL, Kruse NA, Jones I, et al. Specific and nonspecific responses to Plasmodium falciparum blood-stage parasites and observations on the gametocytemia in schoolchildren living in a malaria-endemic area of Mozambique. Am J Trop Med Hyg 1995;52:50-9.

16. Drakeley CJ, Secka I, Correa S, Greenwood BM, Targett GAT. Host haematological factors influencing the transmission of Plasmodium falciparum gametocytes to Anopheles gambiae s.s. mosquitoes. Trop Med Int Health 1999;4:131-8.
17. Osorio L, Ferro BE, Castillo CM. Effects of chloroquine and sufadoxine/pyrimethamine on gametocytes in patients with uncomplicated $P$. falciparum malaria in Colombia. Mem Inst Oswaldo Cruz 2002;97:1221-3.

18. Méndez F, Muñoz A, Carrasquilla G, Jurado D, Arevalo-Herrera M, Cortes J, et al. Determinants of treatment response to sulfadoxine-pyrimethamine and subsequent transmission potential in falciparum malaria. Am J Epidemiol 2002;156:1-9.

19. Dyer M, Day KP. Commitment to gametocytogenesis in Plasmodium falciparum. Parasitol Today 2000;16: 102-7.

20. Mulder B, Tchuinkam T, Dechering K, Verhave JP, Carnavale $\mathrm{P}$, Meuwissen $\mathrm{J}$ et al. Malaria transmission-blocking activity in experimental infections of Anopheles gambiae from naturally infected Plasmodium falciparum gametocyte carriers. Trans $\mathrm{R}$ Soc Trop Med Hyg 1994; 88:121-5.

21. Sokhna CS, Trape JF, Robert V. Gametocitaemia in Senegalese children with uncomplicated falciparum malaria treated with chloroquine, amodiaquine or sulfadoxine + pyrimethamine. Parasite. 2001;8:243-50.

22. http://www.doh.gov.za/issues/malaria/red_reference/ cross_cutting/cross18.pdf (Revisión en agosto de 2003).

23. Blair S, Lacharme L, Carmona J, Tobón A. Resistencia de Plasmodium falciparum a tres fármacos antimaláricos en Turbo (Antioquia, Colombia), 1998. Rev Panam Salud Pública 2001;9:23-9.

24. Blair S, Lacharme L, Carmona J. Resistance of Plasmodium falciparum to antimalarial drugs in Zaragoza (Antioquia, Colombia), 1998. Mem Inst Oswaldo Cruz 2002;97:401-6.

25. Organización Mundial de la Salud/Organización Panamericana de la Salud. Evaluación de la eficacia terapéutica de los medicamentos para el tratamiento del paludismo por Plasmodium falciparum sin complicaciones en las Américas. Washington, D.C., OPS; 1998.

26. Martínez-Bencardino C. Estadística. Bogotá: Ediciones Ecoe; 1993.

27. Graves PM, Carter R, McNeill M. Gametocyte production in cloned lines of Plasmodium falciparum. Am J Trop Med Hyg 1984;33:1045-50.

28. Biswas S. Formation of Plasmodium falciparum gametocytes in vivo and in vitro relates to transmission intensity. Ann Trop Med Parasitol 2000;94:437-46.

29. Montoya L, Maestre A, Carmona J, Lopes D, Do Rosario V, Blair S. Plasmodium falciparum: diversity studies from two Colombian regions with different endemicity. Exp Parasitol 2003;104:14-9. 
30. Smith TG, Walliker D, Ranford-Cartwright LC. Sexual differentiation and sex determination in the Apicomplexa. Trends Parasitol 2002;18:315-22.

31. Christophers SR. The mechanism of immunity against malaria in communities living under hyperendemic conditions. Ind J Med Research 1924;12:273-94.
32. Eichner M, Diebner HH, Molineaux L, Collins WE, Jeffery GM, Dietz K. Genesis, sequestration and survival of Plasmodium falciparum gametocytes: Parameter estimates from fitting a model to malaria therapy data. Trans R Soc Trop Med Hyg 2001;95:497501. 